\title{
A SUBGROUP OF A DIRECT PRODUCT OF FREE GROUPS WHOSE DEHN FUNCTION HAS A CUBIC LOWER BOUND
}

\author{
WILL DISON
}

\begin{abstract}
We establish a cubic lower bound on the Dehn function of a certain finitely presented subgroup of a direct product of 3 free groups.

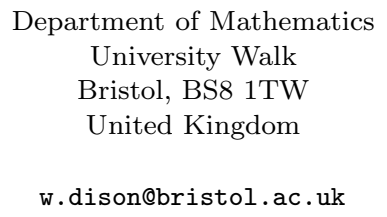

\section{INTRODUCTION}

The collection $\mathcal{S}$ of subgroups of direct products of free groups is surprisingly rich and has been studied by many authors. In the early 1960s Stallings 13. exhibited a subgroup of $\left(F_{2}\right)^{3}$, where $F_{2}$ is the rank 2 free group, as the first known example of a finitely presented group whose third integral homology group is not finitely generated. Bieri 2] demonstrated that Stallings' group belongs to a sequence of groups $\mathrm{SB}_{n} \leq\left(F_{2}\right)^{n}$, the Stallings-Bieri groups, with $\mathrm{SB}_{n}$ being of type $\mathcal{F}_{n-1}$ but not of type $\mathcal{F P}_{n}$. (See [6] for definitions and background concerning finiteness properties of groups.)

In the realm of decision theory, Mihaŭlova [9] and Miller [10] exhibited a finitely generated subgroup of $\left(F_{2}\right)^{2}$ with undecidable conjugacy and membership problems. It is thus seen that even in the 2-factor case fairly wild behaviour is encountered amongst the subgroups of direct products of free groups.

In contrast to this, Baumslag and Roseblade [1] showed that in the 2-factor case this wildness only manifests itself amongst the subgroups which are not finitely presented. They proved that if $G$ is a finitely presented subgroup of $F^{(1)} \times F^{(2)}$, where $F^{(1)}$ and $F^{(2)}$ are free groups, then $G$ is itself virtually a direct product of at most 2 free groups. This work was extended by Bridson, Howie, Miller and Short [5] to an arbitrary number of factors. They proved that if $G$ is a subgroup of a direct product of $n$ free groups and if $G$ enjoys the finiteness property $\mathcal{F} \mathcal{P}_{n}$, then $G$ is virtually a direct product of at most $n$ free groups. Further information on the finiteness properties of the groups in class $\mathcal{S}$ was provided by Meinert [8] who calculated the BNS invariants of direct products of free groups. These invariants determine the finiteness properties of all subgroups lying above the commutator subgroup.

Several authors have investigated the isoperimetric behaviour of the finitely presented groups in $\mathcal{S}$. Elder, Riley, Young and this author [7] have shown that the

2000 Mathematics Subject Classification. 20F65(primary), 20E05, $20 \mathrm{~F} 06$ (secondary). 
Dehn function of Stallings' group $\mathrm{SB}_{3}$ is quadratic. The method espoused by Bridson in [3] proves that the function $n^{3}$ is an upper bound on the Dehn functions of each of the Stallings-Bieri groups. In contrast, there have been no results which give non-trivial lower bounds on the Dehn functions of any groups in $\mathcal{S}$. In other words, until now there has been no finitely presented subgroup of a direct product of free groups whose Dehn function was known to be greater than that of the ambient group. The purpose of this paper is to construct such a subgroup: we exhibit a finitely presented subgroup of $\left(F_{2}\right)^{3}$ whose Dehn function has the function $n^{3}$ as a lower bound.

Let $K$ be the kernel of a homomorphism $\theta:\left(F_{2}\right)^{3} \rightarrow \mathbb{Z}^{2}$ whose restriction to each factor $F_{2}$ is surjective. By Lemma 3.1 below the isomorphism class of $K$ is independent of the homomorphism chosen. Results in [8] show that $K$ is finitely presented.

Theorem 1.1. The Dehn function $\delta$ of $K$ satisfies $\delta(n) \succeq n^{3}$.

Note that Theorem 1.1 makes no reference to a specific presentation of $K$ since, as is well known, the Dehn function of a group is independent (up to $\simeq$-equivalence) of the presentation chosen. We refer the reader to Section 2 for background on Dehn functions, including definitions of the symbols $\succeq$ and $\simeq$.

The organisation of this paper is as follows. Section 2 gives basic definitions concerning Dehn functions, van Kampen diagrams and Cayley complexes. We expect that the reader will already be familiar with these concepts; the purpose of the exposition is principally to introduce notation. In Section 3 we define a class of subgroups of direct products of free groups of which $K$ is a member. The subsequent section gives finite generating sets for those groups in the class which are finitely generated and Section 5 proves a splitting theorem which shows how certain groups in the class decompose as amalgamated products. We then prove in Section 6 how, in certain circumstances, the distortion of the subgroup $H$ in an amalgamated product $\Gamma=G_{1} *_{H} G_{2}$ gives rise to a lower bound on the Dehn function of $\Gamma$. Theorem 1.1 follows as a corollary of this result when applied to the splitting of $K$ given in Section 5 .

\section{Dehn FunCtions}

In this section we recall the basic definitions concerning Dehn functions of finitely presented groups. All of this material is standard. For further background and a more thorough exposition, including proofs, see, for example, [4] or [11].

Given a set $\mathcal{A}$, write $\mathcal{A}^{-1}$ for the set of formal inverses of the elements of $\mathcal{A}$ and write $\mathcal{A}^{ \pm 1}$ for the set $\mathcal{A} \cup \mathcal{A}^{-1}$. Denote by $\mathcal{A}^{ \pm *}$ the free monoid on the set $\mathcal{A}^{ \pm 1}$. We refer to elements of $\mathcal{A}^{ \pm *}$ as words in the letters $\mathcal{A}^{ \pm 1}$ and write $|w|$ for the length of such a word $w$. Given words $w_{1}, w_{2} \in \mathcal{A}^{ \pm *}$ we write $w_{1} \stackrel{\text { free }}{=} w_{2}$ if $w_{1}$ and $w_{2}$ are freely equal and $w_{1} \equiv w_{2}$ if $w_{1}$ and $w_{2}$ are equal as elements of $\mathcal{A}^{ \pm *}$.

Definition 2.1. Let $\mathcal{P}=\langle\mathcal{A} \mid \mathcal{R}\rangle$ be a finite presentation of a group $G$. A word $w \in \mathcal{A}^{ \pm *}$ is said to be null-homotopic over $\mathcal{P}$ if it represents the identity in $G$. A null-P-expression for such a word is a sequence $\left(x_{i}, r_{i}\right)_{i=1}^{m}$ in $\mathcal{A}^{ \pm *} \times \mathcal{R}^{ \pm 1}$ such that

$$
w \stackrel{\text { free }}{=} \prod_{i=1}^{m} x_{i} r_{i} x_{i}^{-1}
$$


Define the area of a null- $\mathcal{P}$-expression to be the integer $m$ and define the $\mathcal{P}$-area of $w$, written $\operatorname{Area}_{\mathcal{P}}(w)$, to be the minimal area taken over all null-P-expressions for $w$.

The Dehn function of the presentation $\mathcal{P}$, written $\delta_{\mathcal{P}}$, is defined to be the function $\mathbb{N} \rightarrow \mathbb{N}$ given by

$$
\delta_{\mathcal{P}}(n)=\max \left\{\operatorname{Area} \mathcal{P}(w): w \in \mathcal{A}^{ \pm *}, w \text { null-homotopic, }|w| \leq n\right\} .
$$

Although the Dehn functions of different finite presentations of a fixed group may differ, their asymptotic behaviour will be the same. This is made precise in the following way.

Definition 2.2. Given functions $f, g: \mathbb{N} \rightarrow \mathbb{N}$, write $f \preceq g$ if there exists a constant $C>0$ such that $f(n) \leq C g(C n+C)+C n+C$ for all $n$. Write $f \simeq g$ if $f \preceq g$ and $g \preceq f$.

Lemma 2.3. If $\mathcal{P}_{1}$ and $\mathcal{P}_{2}$ are finite presentations of the same group then $\delta_{\mathcal{P}_{1}} \simeq$ $\delta_{\mathcal{P}_{2}}$.

For a proof of this standard result see, for example, [4, Proposition 1.3.3].

A useful tool for the study of Dehn functions is a class of objects known as van Kampen diagrams. Roughly speaking, these are planar CW-complexes which portray diagrammatically schemes for reducing null-homotopic words to the identity. Such diagrams, whose definition is recalled below, allow the application of topological methods to the calculation of Dehn functions. For background and further details see, for example, 4, Section 4]. For the definition of a combinatorial CW-complex see, for example, [4, Appendix A].

Definition 2.4. A singular disc diagram $\Delta$ is a finite, planar, contractible combinatorial CW-complex with a specified base vertex $\star$ in its boundary. The area of $\Delta$, written $\operatorname{Area}(\Delta)$, is defined to be the number of 2 -cells of which $\Delta$ is composed. The boundary cycle of $\Delta$ is the edge loop in $\Delta$ which starts at $\star$ and traverses $\partial \Delta$ in the anticlockwise direction. The interior of $\Delta$ consists of a number of disjoint open 2-discs, the closures of which are called the disc components of $\Delta$.

Each 1-cell of $\Delta$ has associated to it two directed edges $\epsilon_{1}$ and $\epsilon_{2}$, with $\epsilon_{1}^{-1}=\epsilon_{2}$. Let DEdge $(\Delta)$ be the set of directed edges of $\Delta$. A labelling of $\Delta$ over a set $\mathcal{A}$ is a map $\lambda$ : DEdge $(\Delta) \rightarrow \mathcal{A}^{ \pm 1}$ such that $\lambda\left(\epsilon^{-1}\right)=\lambda(\epsilon)^{-1}$. This induces a map from the set of edge paths in $\Delta$ to $\mathcal{A}^{ \pm *}$. The boundary label of $\Delta$ is the word in $\mathcal{A}^{ \pm *}$ associated to the boundary cycle.

Let $\mathcal{P}=\langle\mathcal{A} \mid \mathcal{R}\rangle$ be a finite presentation. A $\mathcal{P}$-van Kampen diagram for a word $w \in \mathcal{A}^{ \pm *}$ is a singular disc diagram $\Delta$ labelled over $\mathcal{A}$ with boundary label $w$ and such that for each 2-cell $c$ of $\Delta$ the anticlockwise edge loop given by the attaching map of $c$, starting at some vertex in $\partial c$, is labelled by a word in $\mathcal{R}^{ \pm 1}$.

Lemma 2.5 (Van Kampen's Lemma). A word $w \in \mathcal{A}^{ \pm *}$ is null-homotopic over $\mathcal{P}$ if and only if there exists a $\mathcal{P}$-van Kampen diagram for $w$. In this case the $\mathcal{P}$-area of $w$ is the minimal area over all $\mathcal{P}$-van Kampen diagrams for $w$.

For a proof of this result see, for example, [4, Theorem 4.2.2].

Associated to a presentation $\mathcal{P}=\langle\mathcal{A} \mid \mathcal{R}\rangle$ of a group $G$ there is a standard combinatorial 2-complex $K_{\mathcal{P}}$ with $\pi_{1}\left(K_{\mathcal{P}}\right) \cong G$. The complex $K_{\mathcal{P}}$ is constructed by taking a wedge of copies of $S^{1}$ indexed by the letters in $\mathcal{A}$ and attaching 2-cells indexed by the relations in $\mathcal{R}$. The 2 -cell corresponding to a relation $r \in \mathcal{R}$ has $|r|$ 
edges and is attached by identifying its boundary circuit with the edge path in $K_{\mathcal{P}}^{1}$ along which one reads the word $r$.

The Cayley 2-complex associated to $\mathcal{P}$, denoted by $\operatorname{Cay}^{2}(\mathcal{P})$, is defined to be the universal cover of $K_{\mathcal{P}}$. If one chooses a base vertex of $\operatorname{Cay}^{2}(\mathcal{P})$ to represent the identity element of $G$ then the 1-skeleton of this complex is canonically identified with the Cayley graph of $\mathcal{P}$. Given a $\mathcal{P}$-van Kampen diagram $\Delta$ there is a unique label-preserving combinatorial map from $\Delta$ to $\operatorname{Cay}^{2}(\mathcal{P})$ which maps the base vertex of $\Delta$ to the vertex of $\operatorname{Cay}^{2}(\mathcal{P})$ representing the identity.

\section{A Class of Subgroups of Direct Products of Free Groups}

In this section we introduce a class of subgroups of direct products of free groups of which the group $K$ defined in the introduction will be a member. We first fix some notation which will be used throughout the paper. Given integers $i, m \in \mathbb{N}$, let $F_{m}^{(i)}$ be the rank $m$ free group with basis $e_{1}^{(i)}, \ldots, e_{m}^{(i)}$. Given an integer $r \in \mathbb{N}$, let $\mathbb{Z}^{r}$ be the rank $r$ free abelian group with basis $t_{1}, \ldots, t_{r}$.

Given positive integers $n, m \geq 1$ and $r \leq m$, we wish to define a group $K_{m}^{n}(r)$ to be the kernel of a homomorphism $\theta: F_{m}^{(1)} \times \ldots \times F_{m}^{(n)} \rightarrow \mathbb{Z}^{r}$ whose restriction to each factor $F_{m}^{(i)}$ is surjective. For fixed $n, m$ and $r$, the isomorphism class of the group $K_{m}^{n}(r)$ is, up to an automorphism of the factors of the ambient group $F_{m}^{(1)} \times \ldots \times F_{m}^{(n)}$, independent of the homomorphism $\theta$. This is proved by the following lemma.

Lemma 3.1. Let $F$ be a rank $m$ free group. Given a surjective homomorphism $\phi: F \rightarrow \mathbb{Z}^{r}$, there exists a basis $e_{1}, \ldots, e_{m}$ of $F$ so that

$$
\phi\left(e_{i}\right)= \begin{cases}t_{i} & \text { if } 1 \leq i \leq r \\ 0 & \text { if } r+1 \leq i \leq m .\end{cases}
$$

Proof. The homomorphism $\phi$ factors through the abelianisation homomorphism $\mathrm{Ab}: F \rightarrow A$, where $A$ is the rank $m$ free abelian group $F /[F, F]$, as $\phi=\bar{\phi} \circ \mathrm{Ab}$ for some homomorphism $\bar{\phi}: A \rightarrow \mathbb{Z}^{r}$. Since $\bar{\phi}$ is surjective, $A$ splits as $A_{1} \oplus A_{2}$ where $\bar{\phi}$ is an isomorphism on the first factor and 0 on the second factor. There thus exists a basis $s_{1}, \ldots, s_{m}$ for $A$ so as

$$
\bar{\phi}\left(s_{i}\right)= \begin{cases}t_{i} & \text { if } 1 \leq i \leq r \\ 0 & \text { if } r+1 \leq i \leq m .\end{cases}
$$

We claim that the $s_{i}$ lift under Ab to a basis for $F$. To see this let $f_{1}, \ldots, f_{m}$ be any basis for $F$ and let $\bar{f}_{1}, \ldots, \bar{f}_{m}$ be its image under Ab, a basis for $A$. Let $\rho \in$ Aut $(A)$ be the change of basis isomorphism from $\bar{f}_{1}, \ldots, \bar{f}_{m}$ to $s_{1}, \ldots, s_{m}$. It suffices to show that this lifts under $\mathrm{Ab}$ to an automorphism of $F$. But this is certainly the case since $\operatorname{Aut}(A) \cong G L_{m}(\mathbb{Z})$ is generated by the elementary transformations and each of these obviously lifts to an automorphism.

Definition 3.2. For integers $n, m \geq 1$ and $r \leq m$, define $K_{m}^{n}(r)$ to be the kernel of the homomorphism $\theta: F_{m}^{(1)} \times \ldots \times F_{m}^{(n)} \rightarrow \mathbb{Z}^{r}$ given by

$$
\theta\left(e_{j}^{(i)}\right)= \begin{cases}t_{j} & \text { if } 1 \leq j \leq r \\ 0 & \text { if } r+1 \leq j \leq m .\end{cases}
$$


Note that $K_{2}^{n}(1)$ is the $n^{\text {th }}$ Stallings-Bieri group $\mathrm{SB}_{n}$.

By a result in Section 1.6 of [8], if $r \geq 1$ and $m \geq 2$ then $K_{m}^{n}(r)$ is of type $\mathcal{F}_{n-1}$ but not of type $\mathcal{F P}_{n}$. In particular the group $K \cong K_{2}^{3}(2)$ defined in the introduction is finitely presented.

\section{Generating Sets}

We give finite generating sets for those groups $K_{m}^{n}(r)$ which are finitely generated. We make use of the following notational shorthand: given formal symbols $x$ and $y$, write $[x, y]$ for $x y x^{-1} y^{-1}$ and $x^{y}$ for $y x y^{-1}$.

Proposition 4.1. If $n \geq 2$ then $K_{m}^{n}(r)$ is generated by $S_{1} \cup S_{2} \cup S_{3}$ where

$$
\begin{aligned}
& S_{1}=\left\{e_{i}^{(1)}\left(e_{i}^{(j)}\right)^{-1}: 1 \leq i \leq r, 2 \leq j \leq n\right\}, \\
& S_{2}=\left\{e_{i}^{(j)}: r+1 \leq i \leq m, 1 \leq j \leq n\right\}, \\
& S_{3}=\left\{\left[e_{i}^{(1)}, e_{j}^{(1)}\right]: 1 \leq i<j \leq r\right\} .
\end{aligned}
$$

Proof. Partition $S_{2}$ as $S_{2}^{\prime} \cup S_{2}^{\prime \prime}$ where

$$
S_{2}^{\prime}=\left\{e_{i}^{(j)}: r+1 \leq i \leq m, 2 \leq j \leq n\right\}
$$

and

$$
S_{2}^{\prime \prime}=\left\{e_{i}^{(1)}: r+1 \leq i \leq m\right\} .
$$

Project $K_{m}^{n}(r) \leq F_{m}^{(1)} \times \ldots \times F_{m}^{(n)}$ onto the last $n-1$ factors to give the short exact sequence $1 \rightarrow K_{m}^{1}(r) \rightarrow K_{m}^{n}(r) \rightarrow F_{m}^{(2)} \times \ldots \times F_{m}^{(n)} \rightarrow 1$. Note that $S_{1} \cup S_{2}^{\prime}$ projects to a set of generators for $F_{m}^{(2)} \times \ldots \times F_{m}^{(n)}$ and that $K_{m}^{1}(r)$ is the normal closure in $F_{m}^{(1)}$ of $S_{2}^{\prime \prime} \cup S_{3}$. If $\zeta \in F_{m}^{(1)}$ and $w \equiv w\left(e_{1}^{(1)}, \ldots, e_{m}^{(1)}\right)$ is a word in the generators of $F_{m}^{(1)}$ then

$$
\zeta^{w}=\zeta^{w\left(e_{1}^{(1)}\left(e_{1}^{(2)}\right)^{-1}, \ldots, e_{m}^{(1)}\left(e_{m}^{(2)}\right)^{-1}\right)} .
$$

Thus $S_{1} \cup S_{2}^{\prime} \cup S_{2}^{\prime \prime} \cup S_{3}$ generates $K_{m}^{n}(r)$.

\section{A Splitting Theorem}

The following result gives an amalgamated product decomposition of the groups $K_{m}^{n}(r)$ in the case that $r=m$. Note that with slightly more work one could prove a more general result without this restriction. We introduce the following notation: given a collection of groups $M, L_{1}, \ldots, L_{k}$ with $M \leq L_{i}$ for each $i$, we denote by $*_{i=1}^{k}\left(L_{i} ; M\right)$ the amalgamated product $L_{1} *_{M} \ldots *_{M} L_{k}$.

Theorem 5.1. If $n \geq 2$ and $m \geq 1$ then $K_{m}^{n}(m) \cong *_{k=1}^{m}\left(L_{k} ; M\right)$, where $M=$ $K_{m}^{n-1}(m)$ and, for each $k=1, \ldots, m$, the group $L_{k} \cong K_{m}^{n-1}(m-1)$ is the kernel of the homomorphism

$$
\theta_{k}: F_{m}^{(1)} \times \ldots \times F_{m}^{(n-1)} \rightarrow \mathbb{Z}^{m-1}
$$

given by

$$
\theta_{k}\left(e_{j}^{(i)}\right)= \begin{cases}t_{j} & \text { if } 1 \leq j \leq k-1 \\ 0 & \text { if } j=k \\ t_{j-1} & \text { if } k+1 \leq j \leq m\end{cases}
$$


Proof. Projecting $K_{m}^{n}(m)$ onto the factor $F_{m}^{(n)}$ gives the short exact sequence $1 \rightarrow$ $K_{m}^{n-1}(m) \rightarrow K_{m}^{n}(m) \rightarrow F_{m}^{(n)} \rightarrow 1$. This splits to show that $K_{m}^{n}(m)$ has the structure of an internal semidirect product $M \rtimes \hat{F}_{m}^{(n)}$ where $\hat{F}_{m}^{(n)} \cong F_{m}^{(n)}$ is the subgroup of $F_{m}^{(n-1)} \times F_{m}^{(n)}$ generated by

$$
e_{1}^{(n-1)}\left(e_{1}^{(n)}\right)^{-1}, \ldots, e_{m}^{(n-1)}\left(e_{m}^{(n)}\right)^{-1} .
$$

Since the action by conjugation of $e_{k}^{(n-1)}\left(e_{k}^{(n)}\right)^{-1}$ on $M$ is the same as the action of $e_{k}^{(n-1)}$, we have that

$$
\begin{aligned}
K_{m}^{n}(m) & =M \rtimes \hat{F}_{m}^{(n)} \\
& \cong *_{k=1}^{m}\left(M \rtimes\left\langle e_{k}^{(n-1)}\left(e_{k}^{(n)}\right)^{-1}\right\rangle ; M\right) \\
& \cong *_{k=1}^{m}\left(M \rtimes\left\langle e_{k}^{(n-1)}\right\rangle ; M\right) .
\end{aligned}
$$

Define a homomorphism $p_{k}: F_{m}^{(1)} \times \ldots \times F_{m}^{(n-1)} \rightarrow \mathbb{Z}$ by

$$
p_{k}\left(e_{j}^{(i)}\right)= \begin{cases}1 & \text { if } j=k, \\ 0 & \text { otherwise }\end{cases}
$$

and note that $L_{k} \cap \operatorname{ker} p_{k}$ is the kernel $K_{m}^{n-1}(m)$ of the standard homomorphism $\theta: F_{m}^{(1)} \times \ldots \times F_{m}^{(n-1)} \rightarrow \mathbb{Z}^{m}$ given in Definition 3.2. Considering the restriction of $p_{k}$ to $L_{k}$ gives the short exact sequence $1 \rightarrow K_{m}^{n-1}(m) \rightarrow L_{k} \rightarrow \mathbb{Z} \rightarrow 1$ which demonstrates that $L_{k}=K_{m}^{n-1}(m) \rtimes\left\langle e_{k}^{(n-1)}\right\rangle$.

\section{Dehn Functions of Amalgamated Products}

In this section we will be concerned with finitely presented amalgamated products $\Gamma=G_{1} *_{H} G_{2}$ where $H, G_{1}$ and $G_{2}$ are finitely generated groups and $H$ is proper in each $G_{i}$. Suppose each $G_{i}$ is presented by $\left\langle\mathcal{A}_{i} \mid \mathcal{R}_{i}\right\rangle$, with $\mathcal{A}_{i}$ finite. Note that we are at liberty to choose the $\mathcal{A}_{i}$ so as each $a \in \mathcal{A}_{i}$ represents an element of $G_{i} \backslash H$. Indeed, since $H$ is proper in $G_{i}$ there exists some $a^{\prime} \in \mathcal{A}_{i}$ representing an element of $G_{i} \backslash H$ and we can replace each other element $a \in \mathcal{A}_{i}$ by $a^{\prime} a$ if necessary.

Let $\mathcal{B}$ be a finite generating set for $H$ and for each $b \in \mathcal{B}$ choose words $u_{b} \in \mathcal{A}_{1}^{ \pm *}$ and $v_{b} \in \mathcal{A}_{2}^{ \pm *}$ which equal $b$ in $\Gamma$. Define $\mathcal{E} \subset\left(\mathcal{A}_{1} \cup \mathcal{A}_{2} \cup \mathcal{B}\right)^{ \pm *}$ to be the finite collection of words $\left\{b u_{b}^{-1}, b v_{b}^{-1}: b \in \mathcal{B}\right\}$. Then, since $\Gamma$ is finitely presented, there exist finite subsets $\mathcal{R}_{1}^{\prime} \subseteq \mathcal{R}_{1}$ and $\mathcal{R}_{2}^{\prime} \subseteq \mathcal{R}_{2}$ such that $\Gamma$ is finitely presented by

$$
\mathcal{P}=\left\langle\mathcal{A}_{1}, \mathcal{A}_{2}, \mathcal{B} \mid \mathcal{R}_{1}^{\prime}, \mathcal{R}_{2}^{\prime}, \mathcal{E}\right\rangle
$$

Theorem 6.1. Let $w \in \mathcal{A}_{1}^{ \pm *}$ be a word representing an element $h \in H$ and let $u \in \mathcal{A}_{1}^{ \pm *}$ and $v \in \mathcal{A}_{2}^{ \pm *}$ be words representing elements $\alpha \in G_{1} \backslash H$ and $\beta \in G_{2} \backslash H$ respectively. If $[\alpha, h]=[\beta, h]=1$ then

$$
\operatorname{Area}_{\mathcal{P}}\left(\left[w,(u v)^{n}\right]\right) \geq 2 n \mathrm{~d}_{\mathcal{B}}(1, h)
$$

where $\mathrm{d}_{\mathcal{B}}$ is the word metric on $H$ associated to the generating set $\mathcal{B}$.

Proof. Let $\Delta$ be a $\mathcal{P}$-van Kampen diagram for the null-homotopic word $\left[w,(u v)^{n}\right]$ (see Diagram 11). For each $i=1,2, \ldots, n$, define $p_{i}$ to be the vertex in $\partial \Delta$ such that the anticlockwise path in $\partial \Delta$ from the basepoint around to $p_{i}$ is labelled by the word $w(u v)^{i-1} u$. Similarly define $q_{i}$ to be the vertex in $\partial \Delta$ such that the 


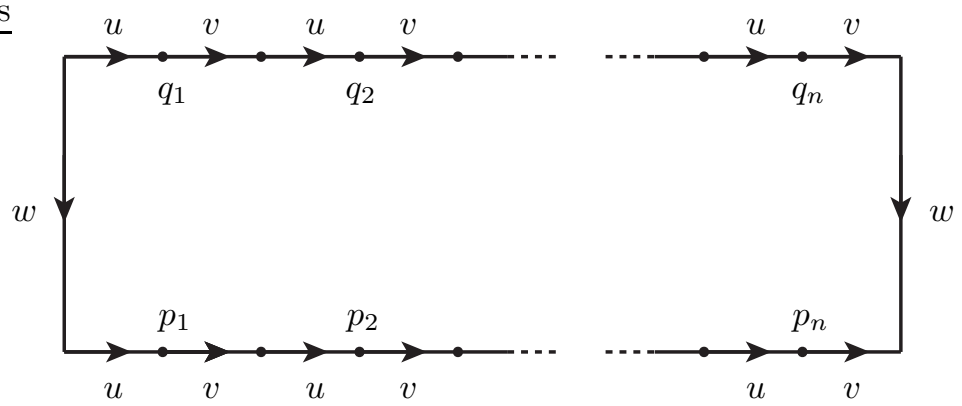

Figure 1. The van Kampen diagram $\Delta$

anticlockwise path in $\partial \Delta$ from the basepoint around to $q_{i}$ is labelled by the word $w(u v)^{n} w^{-1}(u v)^{i-n} v^{-1}$. We will show that for each $i$ there is a $\mathcal{B}$-path (i.e. an edge path in $\Delta$ labelled by a word in the letters $\mathcal{B}$ ) from $p_{i}$ to $q_{i}$.

We assume that the reader is familiar with Bass-Serre theory, as exposited in 12. Let $T$ be the Bass-Serre tree associated to the splitting $G_{1} *_{H} G_{2}$. This consists of an edge $g H$ for each coset $\Gamma / H$ and a vertex $g G_{i}$ for each coset $\Gamma / G_{i}$. The edge $g H$ has initial vertex $g G_{1}$ and terminal vertex $g G_{2}$. We will construct a continuous (but non-combinatorial) map $\Delta \rightarrow T$ as the composition of the natural map $\Delta \rightarrow \operatorname{Cay}^{2}(\mathcal{P})$ with the map $f: \operatorname{Cay}^{2}(\mathcal{P}) \rightarrow T$ defined below.

There is a natural left action of $\Gamma$ on each of $\operatorname{Cay}^{2}(\mathcal{P})$ and $T$ and we construct $f$ to be equivariant with respect to this as follows. Let $m$ be the midpoint of the edge $H$ of $T$ and define $f$ to map the vertex $g \in \operatorname{Cay}^{2}(\mathcal{P})$ to the point $g \cdot m$, the midpoint of the edge $g H$. Define $f$ to map the edge of $\operatorname{Cay}^{2}(\mathcal{P})$ labelled $a \in \mathcal{A}_{i}$ joining vertices $g$ and $g a$ to the geodesic segment joining $g \cdot m$ to $g a \cdot m$. Since $a \notin H$ this segment is an embedded arc of length 1 whose midpoint is the vertex $g G_{i}$. Define $f$ to collapse the edge in $\operatorname{Cay}^{2}(\mathcal{P})$ labelled $b \in \mathcal{B}$ joining vertices $g$ and $g b$ to the point $g \cdot m=g b \cdot m$. This is well defined since $g H=g b H$. This completes the definition of $f$ on the 1-skeleton of $\Delta$; we now extend $f$ over the 2-skeleton.

Let $c$ be a 2-cell in $\operatorname{Cay}^{2}(\mathcal{P})$ and let $g$ be some vertex in its boundary. Assume that $c$ is metrised so as to be convex and let $l$ be some point in its interior. The form of the relations in $\mathcal{P}$ ensures that the boundary label of $c$ is a word in the letters $\mathcal{A}_{i} \cup \mathcal{B}$ for some $i$ and so every vertex in $\partial c$ is labelled $g g^{\prime}$ for some $g^{\prime} \in G_{i}$. Thus $f$ as so far defined maps $\partial c$ into the ball of radius $1 / 2$ centred on the vertex $g G_{i}$; we extend $f$ to the interior of $c$ by defining it to map the geodesic segment $[l, p]$, where $p \in \partial c$, to the geodesic segment $\left[g G_{i}, f(p)\right]$. This is independent of the vertex $g \in \partial c$ chosen and makes $f$ continuous since geodesics in a tree vary continuously with their endpoints. We now define $\bar{f}: \Delta \rightarrow T$ to be the map given by composing $f$ with the label-preserving map $\Delta \rightarrow \operatorname{Cay}^{2}(\mathcal{P})$ which sends the basepoint of $\Delta$ to the vertex $1 \in \operatorname{Cay}^{2}(\mathcal{P})$.

Since $w$ commutes with $u$ and $v$ we have that $\bar{f}\left(p_{i}\right)=w(u v)^{i-1} u \cdot m=(u v)^{i-1} u \cdot m=$ $\bar{f}\left(q_{i}\right)$; define $S$ to be the preimage under $\bar{f}$ of this point. By construction, the image of the interior of each 2-cell in $\Delta$ and the image of the interior of each $\mathcal{A}_{i}$-edge is disjoint from $\bar{f}\left(p_{i}\right)$. Thus $S$ consists of vertices and $\mathcal{B}$-edges and so finding a $\mathcal{B}$-path from $p_{i}$ to $q_{i}$ reduces to finding a path in $S$ connecting these vertices. Let $s_{i}$ and $t_{i}$ be the vertices of $\partial \Delta$ immediately preceding and succeeding $p_{i}$ in the 
boundary cycle. Unless $h=1$, in which case the theorem is trivial, the form of the word $\left[w,(u v)^{n}\right]$, together with the normal form theorem for amalgamated products, implies that all the vertices $p_{i}, s_{i}$ and $t_{i}$ lie in the boundary of the same disc component $D$ of $\Delta$. Furthermore, since $u$ and $v$ are words in the letters $\mathcal{A}_{1}$ and $\mathcal{A}_{2}$ respectively, the points $f\left(s_{i}\right)$ and $f\left(t_{i}\right)$ are separated in $T$ by $f\left(p_{i}\right)$. Thus $s_{i}$ and $t_{i}$ are separated in $D$ by $S$ and so there exists an edge path $\gamma_{i}$ in $S$ from $p_{i}$ to some other vertex $r_{i} \in \partial D$. Since $\gamma_{i}$ is a $\mathcal{B}$-path it follows that the word labelling the sub-arc of the boundary cycle of $\Delta$ from $p_{i}$ to $r_{i}$ represents an element of $H$, and, by considering subwords of $\left[w,(u v)^{n}\right]$, we see that the only possibility is that $r_{i}=q_{i}$. Thus, for each $i=1, \ldots, n$, the path $\gamma_{i}$ gives the required $\mathcal{B}$-path connecting $p_{i}$ to $q_{i}$. We choose each $\gamma_{i}$ to contain no repeated edges.

For $i \neq j$, the two paths $\gamma_{i}$ and $\gamma_{j}$ are disjoint, since if they intersected there would be a $\mathcal{B}$-path joining $p_{i}$ to $p_{j}$ and thus the word labelling the subarc of the boundary cycle from $p_{i}$ to $p_{j}$ would represent an element of $H$. Observe that no two edges in any of the paths $\gamma_{1}, \ldots, \gamma_{n}$ lie in the boundary of the same 2-cell in $\Delta$ since each relation in $\mathcal{P}$ contains at most one occurrence of a letter in $\mathcal{B}$. Because the word labelling $\partial \Delta$ contains no occurrences of a letter in $\mathcal{B}$ the interior of each edge of a path $\gamma_{i}$ lies in the interior of $\Delta$ and thus in the boundary of two distinct 2cells. Since each path $\gamma_{i}$ contains no repeated edges we therefore obtain the bound $\operatorname{Area}(\Delta) \geq \sum_{i=1}^{n} 2\left|\gamma_{i}\right|$. But the word labelling each $\gamma_{i}$ is equal to $h$ in $\Gamma$ and so the length of $\gamma_{i}$ is at least $\mathrm{d}_{\mathcal{B}}(1, h)$ whence we obtain the required inequality.

We are now in a position to prove the main theorem.

Proof of Theorem 1.1. To avoid excessive superscripts we change notation and write $x_{i}, y_{i}$ for the generators of $F_{2}^{(i)}, i=1,2$.

By Proposition 4.1 and Theorem 5.1 we have that $K \cong K_{2}^{3}(2) \cong L_{1} *_{M} L_{2}$ where, as subgroups of $F_{2}^{(1)} \times F_{2}^{(2)}, L_{1}=K_{2}^{2}(1)$ is generated by $\mathcal{A}_{1}=\left\{x_{1} x_{2}^{-1}, y_{1}, y_{2}\right\}$, $L_{2} \cong K_{2}^{2}(1)$ is generated by $\mathcal{A}_{2}=\left\{x_{1}, x_{2}, y_{1} y_{2}^{-1}\right\}$ and $M=K_{2}^{2}(2)$ is generated by $\mathcal{B}=\left\{x_{1} x_{2}^{-1}, y_{1} y_{2}^{-1},\left[x_{1}, y_{1}\right]\right\}$. To obtain the generating set for $L_{2}$ we have here implicitly used the automorphism of $F_{2}^{(1)} \times F_{2}^{(2)}$ which interchanges $x_{i}$ with $y_{i}$ and realises the isomorphism between $L_{2}$ and $K_{2}^{2}(1)$.

For $n \in \mathbb{N}$, define $h_{n}$ to be the element $\left[x_{1}^{n}, y_{1}^{n}\right] \in K_{2}^{2}(2)$ and define $w_{n}$ to be the word $\left[\left(x_{1} x_{2}^{-1}\right)^{n}, y_{1}^{n}\right] \in \mathcal{A}_{1}^{ \pm *}$ representing $h_{n}$. Note that $h_{n}$ commutes with both $y_{2} \in \mathcal{A}_{1}$ and $x_{2} \in \mathcal{A}_{2}$ so by Theorem 6.1 the word $\left[w_{n},\left(y_{2} x_{2}\right)^{n}\right]$, which has length $16 n$, has area at least $2 n d_{\mathcal{B}}\left(1, h_{n}\right)$. We claim that $d_{\mathcal{B}}\left(1, h_{n}\right) \geq n^{2}$.

Suppose that in $F_{2}^{(1)} \times F_{2}^{(2)}$ the element $h_{n}$ is represented by a word $w \equiv$ $w\left(x_{1} x_{2}^{-1}, y_{1} y_{2}^{-1},\left[x_{1}, y_{1}\right]\right)$ in the generators $\mathcal{B}$. Let $k$ be the number of occurrences of the third variable in the word $w$. We will show that $k \geq n^{2}$.

Observe that, as a group element, the word $w\left(x_{1} x_{2}^{-1}, y_{1} y_{2}^{-1},\left[x_{1}, y_{1}\right]\right)$ is equal to the word $w\left(x_{1}, y_{1},\left[x_{1}, y_{1}\right]\right) w\left(x_{2}^{-1}, y_{2}^{-1}, 1\right)$. Thus we have that $\left[x_{1}^{n}, y_{1}^{n}\right]$ is freely equal to $w\left(x_{1}, y_{1},\left[x_{1}, y_{1}\right]\right)$ and that $w\left(x_{2}^{-1}, y_{2}^{-1}, 1\right)$, and thus $w\left(x_{1}, y_{1}, 1\right)$, is freely equal to the empty word. It follows that $\left[x_{1}^{n}, y_{1}^{n}\right]$ can be converted to the empty word by free expansions, free contractions and deletion of $k$ subwords $\left[x_{1}, y_{1}\right]$. Hence $\left[x_{1}^{n}, y_{1}^{n}\right]$ is a null-homotopic word over the presentation $\mathcal{P}=\left\langle x_{1}, y_{1} \mid\left[x_{1}, y_{1}\right]\right\rangle$ with $\mathcal{P}$-area at most $k$. But $\mathcal{P}$ presents the rank 2 free abelian group, and basic results on Dehn functions give that $\left[x_{1}^{n}, y_{1}^{n}\right]$ has area $n^{2}$ over this presentation. Thus $k \geq n^{2}$. 
Note that the above proof also shows that $K_{2}^{2}(2)$ has at least quadratic distortion in each of $K_{2}^{2}(1)$ and $F_{2}^{(1)} \times F_{2}^{(2)}$. In fact it can be shown that the distortion is precisely quadratic.

Acknowledgements. I would like to thank my thesis advisor, Martin Bridson, for his many helpful comments made during the preparation of this article.

\section{REFERENCES}

[1] G. Baumslag and J. E. Roseblade. Subgroups of direct products of free groups. J. London Math. Soc. (2), 30(1):44-52, 1984

[2] R. Bieri. Homological dimension of discrete groups. Mathematics Department, Queen Mary College, London, 1976. Queen Mary College Mathematics Notes.

[3] M. R. Bridson. Doubles, finiteness properties of groups, and quadratic isoperimetric inequalities. J. Algebra, 214(2):652-667, 1999.

[4] M. R. Bridson. The geometry of the word problem. In Invitations to geometry and topology, volume 7 of Oxf. Grad. Texts Math., pages 29-91. Oxford Univ. Press, Oxford, 2002.

[5] M. R. Bridson, J. Howie, C. F. Miller, III, and H. Short. The subgroups of direct products of surface groups. Geom. Dedicata, 92:95-103, 2002. Dedicated to John Stallings on the occasion of his 65 th birthday.

[6] K. S. Brown. Cohomology of groups, volume 87 of Graduate Texts in Mathematics. SpringerVerlag, New York, 1982.

[7] W. Dison, M. Elder, T. Riley, and R. Young. The Dehn function of Stallings' group. arXiv:0712.3877 1 .

[8] H. Meinert. The geometric invariants of direct products of virtually free groups. Comment. Math. Helv., 69(1):39-48, 1994.

[9] K. A. Mihallova. The occurrence problem for direct products of groups. Dokl. Akad. Nauk SSSR, 119:1103-1105, 1958.

[10] C. F. Miller, III. On group-theoretic decision problems and their classification. Princeton University Press, Princeton, N.J., 1971. Annals of Mathematics Studies, No. 68.

[11] T. Riley. Filling functions. In The Geometry of the Word Problem for Finitely Generated Groups, Advanced Courses in Mathematics. CRM Barcelona, pages 81-151. Birkhäuser Verlag, Basel, 2007.

[12] J.-P. Serre. Trees. Springer-Verlag, Berlin, 1980. Translated from the French by John Stillwell.

[13] J. Stallings. A finitely presented group whose 3-dimensional integral homology is not finitely generated. Amer. J. Math., 85:541-543, 1963.

Will Dison, Department of Mathematics, University Walk, Bristol, BS8 1TW, United KINGDOM

E-mail address: w.dison@bristol.ac.uk 